\title{
Fungal artificial chromosomes for mining of the fungal secondary metabolome
}

\author{
Jin Woo Bok ${ }^{1 \dagger}$, Rosa Ye ${ }^{2,4+}$, Kenneth D Clevenger ${ }^{3 \dagger}$, David Mead ${ }^{4}$, Megan Wagner ${ }^{4}$, Amanda Krerowicz ${ }^{4}$, \\ Jessica C Albright ${ }^{5}$, Anthony W Goering ${ }^{6}$, Paul M Thomas ${ }^{3,6}$, Neil L Kelleher ${ }^{3,5,6^{*}}$, Nancy P Keller ${ }^{1^{*}}$ \\ and Chengcang $\mathrm{C} \mathrm{Wu} \mathrm{U}^{2,4^{*}}$
}

\begin{abstract}
Background: With thousands of fungal genomes being sequenced, each genome containing up to 70 secondary metabolite (SM) clusters 30-80 kb in size, breakthrough techniques are needed to characterize this SM wealth.

Results: Here we describe a novel system-level methodology for unbiased cloning of intact large SM clusters from a single fungal genome for one-step transformation and expression in a model host. All 56 intact SM clusters from Aspergillus terreus were individually captured in self-replicating fungal artificial chromosomes (FACs) containing both the E. coli F replicon and an Aspergillus autonomously replicating sequence (AMA1). Candidate FACs were successfully shuttled between E. coli and the heterologous expression host A. nidulans. As proof-of-concept, an A. nidulans FAC strain was characterized in a novel liquid chromatography-high resolution mass spectrometry (LC-HRMS) and data analysis pipeline, leading to the discovery of the A. terreus astechrome biosynthetic machinery.
\end{abstract}

Conclusion: The method we present can be used to capture the entire set of intact SM gene clusters and/or pathways from fungal species for heterologous expression in A. nidulans and natural product discovery.

Keywords: Fungal artificial chromosome (FAC), Functional genomics, Secondary metabolite (SM) gene clusters, Natural product discovery

\section{Background}

Secondary metabolites (SMs), also known as natural products, are a structurally diverse group of compounds with varied and important biological activities. Fungi are prolific producers of these compounds, which can be classified as polyketide, non-ribosomal peptide, terpene or molecules of mixed heritage (e.g. polyketide-nonribosomal peptide hybrids). Well-known fungal secondary metabolites include the antibiotic penicillin from Penicillium chrysogenum, the immunosuppressant cyclosporine from Tolypocladium inflatum, and the cholesterollowering agent mevinolin (a.k.a. lovastatin) from $A$. terreus. With over 5 million predicted fungal species [1] and

\footnotetext{
*Correspondence: n-kelleher@northwestern.edu; npkeller@wisc.edu; cwu@intactgenomics.com

${ }^{\dagger}$ Equal contributors

${ }^{3}$ Proteomics Center of Excellence, Northwestern University, Evanston, IL, USA 'Department of Medical Microbiology and Immunology and Bacteriology, University of Wisconsin at Madison, Madison, WI, USA

${ }^{2}$ Intact Genomics, Inc., St Louis, MO, USA

Full list of author information is available at the end of the article
}

dozens of secondary metabolite (SM) clusters per species [2], the number of yet undiscovered SMs is quite large. Indeed, recognition of SM clusters and other valuable attributes within the fungal genome led to the DOE Joint Genome Institute's 1000 Fungal Genomes large-scale sequencing project [3].

Despite the abundance of available fungal genetic sequence data highlighting an enormous abundance of SM clusters, many remain 'silent' in laboratory growth conditions and require genetic manipulation to be expressed. Several approaches have been taken to 'turn-on' SM clusters with some success. These include over-expressing cluster specific transcription factors or enzymatic genes, deleting or over-expressing chromatin modifying genes, over-expressing trans-acting activators or deleting transacting inhibitors [4-7]. These molecular machinations, however, only work for genetically amenable fungi of which there are remarkably few. This latter point has led to the use of SM workhorses, most commonly members 
of the genus Aspergillus or yeast, for expression of heterologous SM genes [8-12].

Expression of a heterologous cluster in fungi is one approach to identify the encoded SM and the biosynthetic genes responsible for its biosynthesis, but this is not a trivial undertaking. Recently, this approach has been reported for synthesis of the A. terreus-encoded compounds geodin and asperfuranone using $A$. nidulans as the heterologous host $[9,10]$. A. nidulans was also used to heterologously express a dermatophyte-derived gene cluster responsible for the synthesis of neosartoricin B [12]. These studies utilized yeast recombinatory plasmids, multiple fusion PCRs and numerous transformation events to stitch together and insert individual genes to create a single full length cluster in A. nidulans. These technologies require considerable effort and time to express just one heterologous cluster and have been limited in the size of the inserted cluster.

Bacterial artificial chromosomes (BACs) have been widely used for genomic DNA sequencing, positional cloning, and mapping in prokaryotes and eukaryotes including filamentous fungi [13-17]. Although large-insert DNA systems have also been applied for heterologous expression of microbial natural product biosynthetic pathways and metagenomic studies, there has been limited success reported due to technological challenges [18-20]. The challenges include but are not limited to: 1) DNA cloning bias; 2) small DNA insert size; 3) lack of advanced heterologous expression hosts and 4) insufficient high-resolution chemical and data analysis pipelines. Here we address all of these limitations through creation of a novel Aspergillus/E. coli shuttle fungal artificial chromosome (FAC) expression vector by utilizing unbiased Random Shear BAC technology [21] coupled with an autonomous fungal replicating element AMA1 [22], to expression of FACs in A. nidulans, to characterization of FAC SMs using state-of-art liquid chromatography-high resolution mass spectrometry (LC-HRMS) and a chemoinformatic analysis pipeline. We present the heretofore undiscovered $A$. terreus astechrome biosynthetic machineries as proof-of-concept of our FAC SM methodology.

\section{Results}

Construction of unbiased shuttle BAC library of $A$. terreus DNA and heterologous expression of SM clusters as FACs

\section{in $A$. nidulans}

To develop a fungal artificial chromosome (FAC) system, we used unbiased Random Shear BACs as the basis for our technology as BAC inserts can reach up to $300 \mathrm{~kb}$, and Random Shear BAC cloning generates even coverage of a fungal genome for the selection of all SM clusters within the genome $[21,23]$. The Lucigen pSMART BAC vector was modified to operate as a shuttle vector between $E$. coli and $A$. nidulans. The AMA1 DNA fragment, previously identified as an autonomously replicating DNA fragment from $A$. nidulans [22], was incorporated into pSMART BAC to create pSMART-BAC pyrGAMA1-4, or shuttle BAC vectors, and tested for autonomous replication in $A$. nidulans as FACs (Additional file 1: Figure S1 a, b).

A. terreus was selected for shuttle BAC DNA library construction because it has a fully sequenced genome containing 56 annotated SM gene clusters [24]. High molecular weight genomic DNA was prepared from $A$. terreus and construction of the unbiased BAC library resulted in $\sim 20 \mathrm{x}$ genome coverage of the A. terreus genome, or a total of 7,680 BAC clones with an average insert size of $100 \mathrm{~kb}$ (Additional file 1: Figure S2a, b). The BAC library was arrayed into 384-well plates and both ends of 3,840 BAC clones were sequenced. Sequence alignment of these end sequences with the $A$. terreus reference genome was used to identify SM-BAC clones or candidate FACs containing all 56 SM gene clusters (Additional file 2: Table S1). Fifteen FACs (ranging from 70 to $150 \mathrm{~kb}$ in size) were selected for heterologous expression and analysis through transformation into $A$. nidulans (Table 1 ). All were successfully transformed into $A$. nidulans. To validate the shuttle function of FACs, we also extracted five of the 15 FAC DNAs from transformed $A$. nidulans strains and successfully transformed FAC DNA back into E. coli (Figure 1, Additional file 1: Figure S3). This was the first demonstration of the capability of AMA1 in supporting

Table 1 Fifteen FACs chosen to transform into A. nidulans

\begin{tabular}{|c|c|c|c|c|}
\hline $\begin{array}{l}\text { FAC } \\
\text { name }\end{array}$ & AT cluster(s)* & PCR & $\mathrm{TX} / \mu \mathrm{g} F A C^{* *}$ & Fungal strain \\
\hline $4 \mathrm{G} 11$ & 21 & All & 123 & TJW152 \\
\hline $6 J 7$ & 44 & All & 94 & TJW153 \\
\hline 8J11 & 52 & One end only & 36 & TJW154 \\
\hline 903 & 30 & All & 43 & TJW155 \\
\hline 4012 & 11 & All & 58 & TJW156 \\
\hline $6 \mathrm{H} 10$ & 6 & All & 60 & TJW157 \\
\hline $8 \mathrm{~K} 17$ & 19 & All & 139 & TJW158 \\
\hline $5 \mathrm{~N} 15$ & 39 & All & 24 & TJW159 \\
\hline 7019 & 38 & All & 18 & TJW160 \\
\hline 9F18 & 55 & All & 57 & TJW161 \\
\hline $6 C 13$ & 41 & All & 94 & TJW162 \\
\hline 7P13 & 50 & All & 29 & TJW163 \\
\hline 9D19 & 23 & All & 80 & TJW164 \\
\hline $9 A 23$ & 25 , partial 26 & All & 26 & TJW165 \\
\hline $7 \mathrm{~A} 10$ & 56 & All & 45 & TJW166 \\
\hline Empty & No & & 432 & TJW167 \\
\hline
\end{tabular}

The $A$. terreus clusters they contain are indicated as well as PCR results denoting if all or part of the cluster was confirmed in the FAC clone. ${ }^{*} \mathrm{AT}=A$. terreus, ${ }^{* *} \mathrm{TX}=$ transformants. 


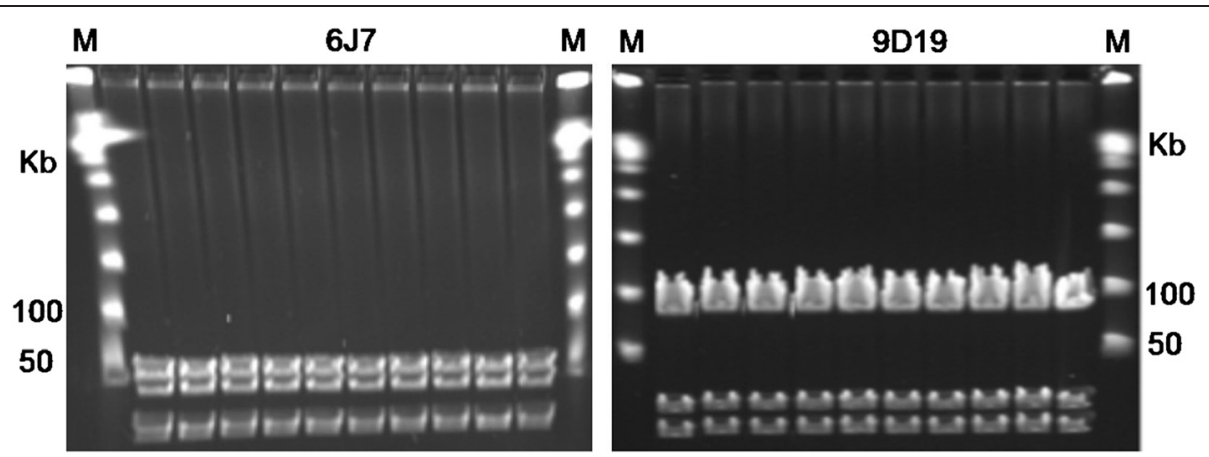

Figure 1 The CHEF gels of E. coli-Aspergillus shuttle FACs: $6 J 7$ (cluster 44, 112 kb) and 9D19 (cluster 23, 100 kb) that were successfully transferred from transformed strains of $A$. nidulans back into $E$. coli. The first and last lanes (M) are DNA Lambda ladder Markers, the $2^{\text {nd }}$ lane on the left hand side of the gels is the control FAC used to transform A. nidulans, and all of other lanes are randomly selected FAC clones recovered. All control and recovered FACs were digested with Notl.

autonomous replication (FAC) of large DNA constructs greater than $100 \mathrm{~kb}$ in A. nidulans.

\section{LC-HRMS linked SM library screening}

Upon confirmation that $A$. nidulans faithfully replicated FAC DNA, A. nidulans FAC 6J7 strain was selected for initial proof-of-concept experiments, as it contained a cluster highly homologous to the recently characterized hexadehydroastechrome cluster in A. fumigatus [25]. FAC 6J7 contains seven out of the eight genes found in the corresponding A. fumigatus cluster (Figure 2a). The gene, has $G$, not present in the $A$. terreus cluster, encodes

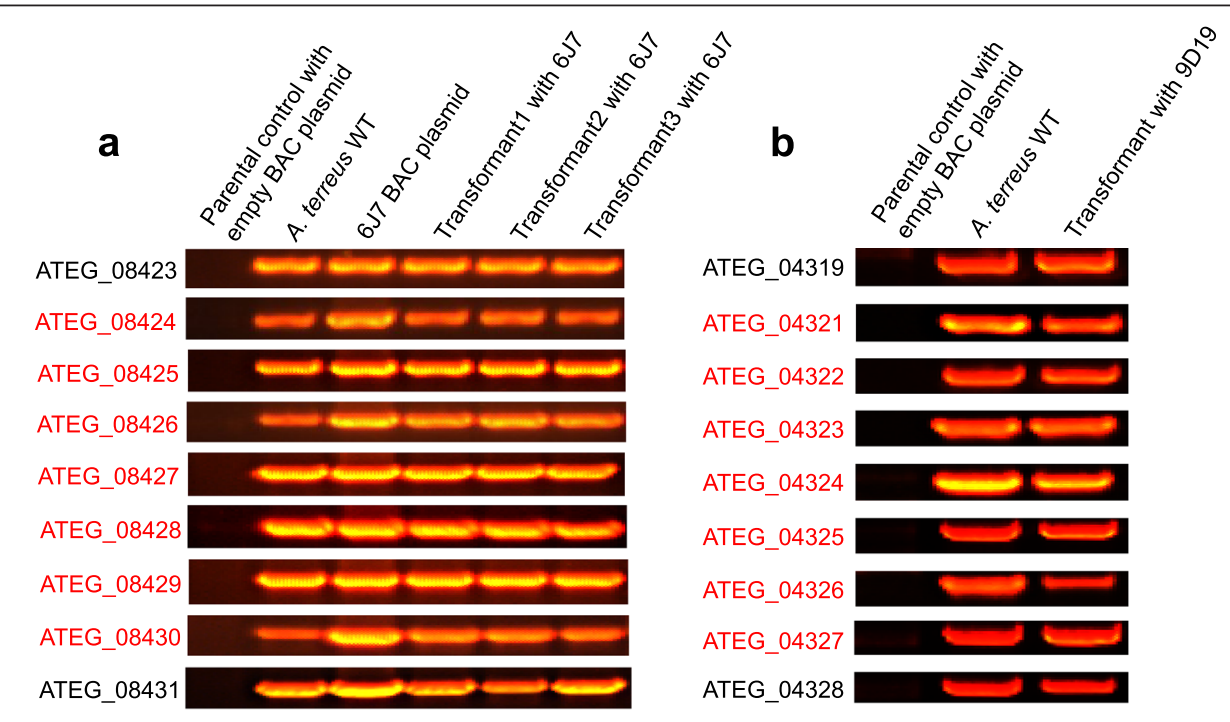

C

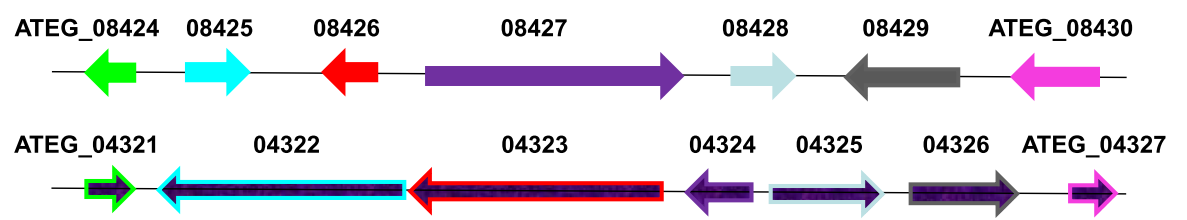

Figure 2 FAC transformants contain intact gene clusters confirmed by PCR. Panel a: Astechrome gene cluster (6J7) is present in A. nidulans transformed with FAC as examined by PCR. The seven astechrome genes (red) are not present in wild type A. nidulans but are in A. terreus and all A. nidulans 6J7 transformants. Panel b: Putative gene cluster 23 (9D19) is present in A. nidulans transformed with FAC 9D19 as examined by PCR. The seven cluster genes (red) are not present in wild type A. nidulans but are in A. terreus and A. nidulans 9D19 transformant. All of primers are listed in Additional file 2: Table S1. Panel c shows both Astechrome gene cluster (6J7) on the top and the cluster 23 (9D19) on the bottom. 
for an FAD binding protein responsible for converting a prenyl to a methylbutadienyl side chain to produce hexadehydroastechrome from astechrome. FAC 6J7 metabolites were identified by analyzing organic extracts of the A. nidulans FAC 6J7 transformant and control A. nidulans using LC-HRMS. Following data acquisition, Sieve software was used for component detection and relative quantitation. When comparing FAC 6J7 extracts to control sample extracts (wild type and other FAC strains), a compound that was present only in the FAC 6J7 extract (Figure $3 \mathrm{~b}$ ) was identified as terezine $\mathrm{D}$ by both accurate mass (0.3 part-per-million error) and tandem mass spectrometry (MS/MS or $\mathrm{MS}^{2}$ ) (Figure 3a,c). Terezine D is a stable intermediate of astechrome biosynthesis [26].

\section{Discussion}

There is an urgent need for new therapeutic agents to combat rapidly-emerging multiple drug resistant (MDR) and pan-resistant pathogens such as methicillin resistant Staphylococcus aureus (MRSA) and Acinetobacter baumanii. Filamentous fungi are prolific producers of SMs and have historically been a rich source of lead compounds for the pharmaceutical industry. Genomic sequencing data confirms that fungi contain a far greater biosynthetic capacity than has been realized to date, and thus fungi should continue to be viewed as important reservoirs for novel bioactive compounds [27-33]. In fact the number of SM cluster sequences available for characterization far outstrips our current ability to characterize each cluster. To address this post-genomic SM characterization gridlock, in this report we have demonstrated a new technology that generates a whole genome SM FAC library for expression in suitable host systems and characterization in high-throughput chemical analysis pipelines. An overview of this technology is presented in Figure 4.

To date, there does not exist an efficient heterologous expression system to cover entire SM clusters (30 $80 \mathrm{~kb}$ ) in a single cloning step. BAC vectors have been widely used for cloning large DNA fragments but there is no successful report of heterologous expression of fungal SM clusters. A previous attempt to introduce up to $75 \mathrm{~Kb}$ of fungal DNA into Fusarium oxysporum and A. awamori using an Agrobacterium tumefaciens transformation system yielded few transformants with large

\section{a Structure of Terezine $\mathrm{D}$ ion}<smiles>C=C1NC(Cc2c[nH]c3c(CC=C(C)C)cccc23)C(=O)NC1COc1ccccc1</smiles>

\section{b Selected Ion Chromatogram}

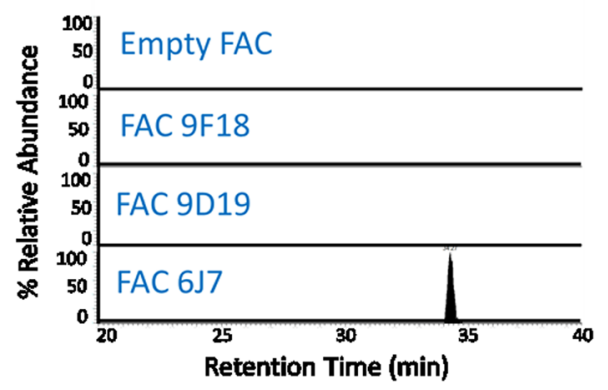

\section{C $\mathrm{MS}^{2}$ of 326.1 lon}

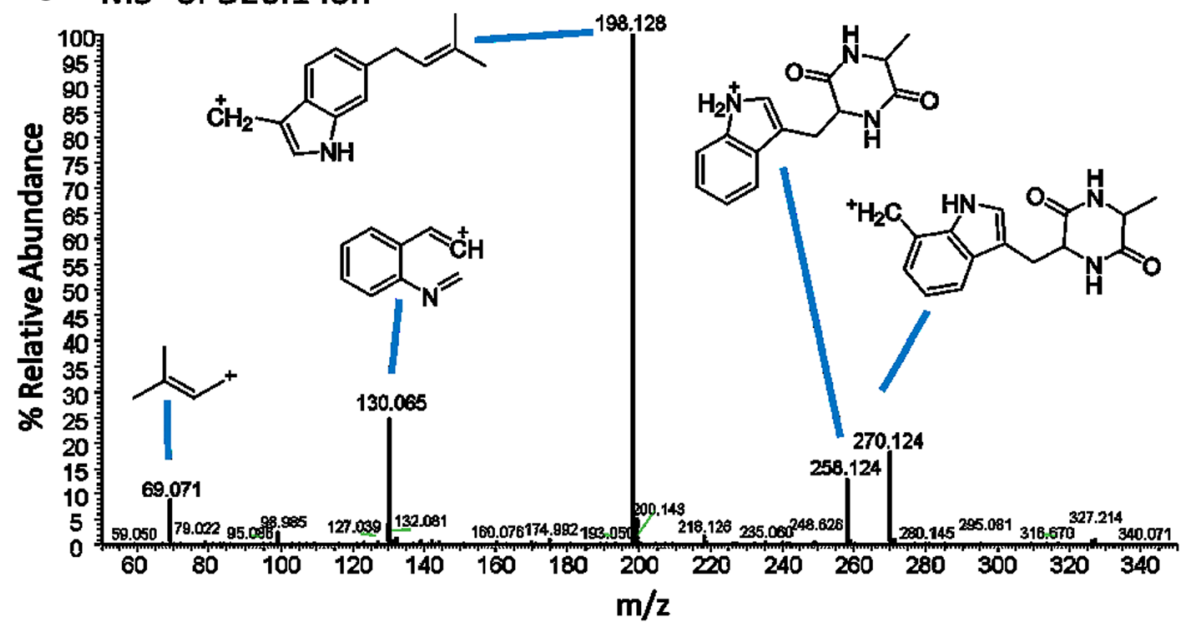

Figure 3 Identification of astechrome biosynthetic precursor, terezine D. (a) A compound was identified in FAC 6J7 cell extracts with exact mass matching the theoretical exact mass of terezine D, the predicted product of FAC 6J7. (b) Selected ion chromatograms of lysates from cells containing FAC 6J7 and all other analyzed FACs show that the compound of interest is unique to the FAC 6J7 expressing strain. (c) Analysis of MS ${ }^{2}$ fragmentation data for the identified compound lead to identification of predicted fragments of terezine $D$, confirming the compound's identity. 


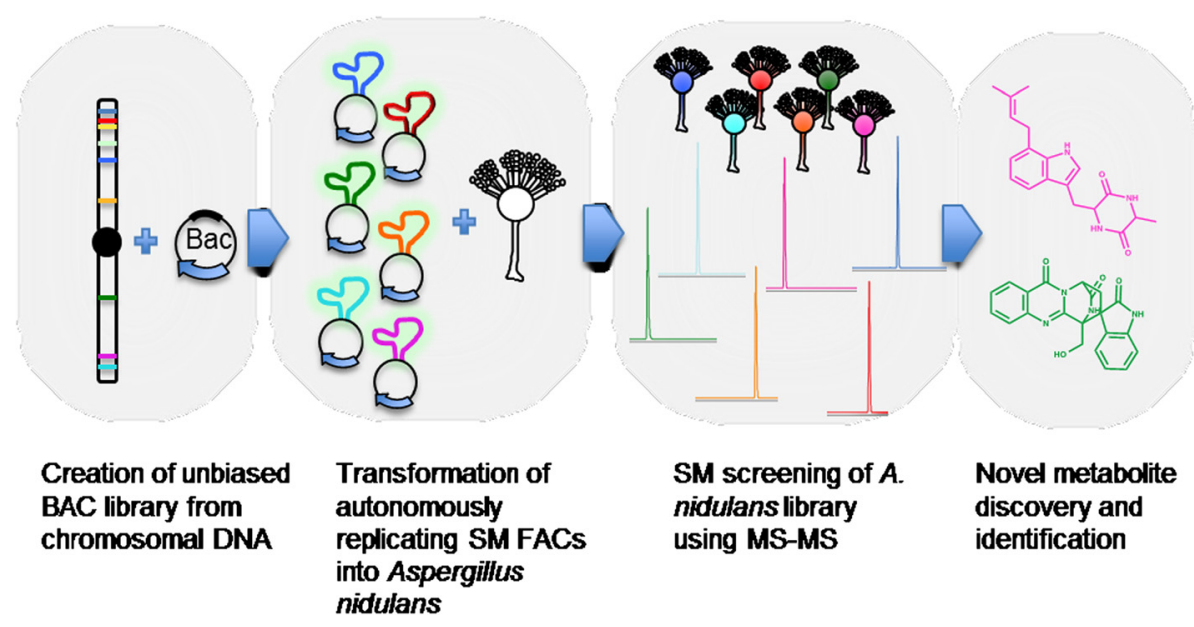

Figure 4 A diagram shows the workflow of cryptic SM production. High molecular weight genomic DNA from A. terreus was mechanically sheared and cloned into shuttle AMAI-BAC (or FAC) clones, SM FACs were selected and transformed into A. nidulans, transformants screened for metabolites followed by compound identification and structure elucidation.

DNA inserts and furthermore, no attempts to examine stability of heterologous DNA, let alone expression, were made [34]. In order to build high quality shuttle BAC libraries for entire SM gene clusters, the cloning methods and vectors used are of extreme importance. The bias introduced by partial restriction digestion of genomic DNA results in various regions being highly under-represented, over-represented, or missing for all eukaryotic multicellular genomes studied, including Arabidopsis, Drosophila, rice, mouse, fungi and humans. The bias is most evident in certain regions of genomic DNA that contain highly repetitive sequences, such as centromeres and telomeres [21]. As a result, numerous clone gaps can be impossible to close, even with multiple biased partial digestion libraries and up to $50 \mathrm{x}$ coverage, thus dramatically increasing finishing costs. Fungi are known to contain many SM clusters in telomeric and subtelomeric regions of the genome $[7,24]$ and from our BAC end sequencing and reference genome alignment, we found that at least 10 of $56 \mathrm{SM}$ clusters of $A$. terreus are located near telomeres and some telomeric sequences are still not complete in the whole genome sequence database (data not shown). We successfully overcame this potential bias in conventional BAC library construction through the introduction of randomly sheared genomic DNA into the FAC vectors.

To improve transformation yield and subsequent expression level of the gene clusters in a heterologous system, the BAC vector was modified into a FAC vector by inserting a fungal self-replicating element. The AMA1 sequence from $A$. nidulans is known to increase transformation efficiency up to 2000-fold compared to a traditional integrating plasmid (reviewed in [22]). The AMA1 sequence was also shown to be fully or partially functional in other filamentous fungal systems including $A$. fumigatus resulting in 10-30 copy numbers per cell and increased gene expression [22,35-40]. By introducing the AMA1 element in a BAC vector, thus creating the FAC system, we were able to introduce and express at least $150 \mathrm{~kb}$ heterologous DNA in $A$. nidulans. Strictly the concept of BAC itself is truly an F-plasmid, not a 'bacterial artificial chromosome', FAC can be justified similarly as BAC with the capability of shuttling and maintaining large DNA and potential wide applications. In addition, FAC, a shuttle BAC vector, is capable of cloning $>300 \mathrm{~kb}$ insert DNA in E. coli [41]; it will be interesting to see the size limitation and stability of more FACs in A. nidulans as compared with the yeast artificial chromosome (YAC) in Saccharomyces cerevisiae [42].

The entire A. terreus SM genome was captured in our FAC library with 56 SM clusters (Additional file 2: Table $\mathrm{S} 1$ ). The availability of such a library is powerful, allowing us to swiftly screen the host recipient for individual SM activities. The host recipient is critical for transformation and expression properties and we found the genetic model A. nidulans to be an efficient host (see Methods for FAC transformation optimization). As a first assessment of FAC transformation, 15 FACs were successfully expressed in $A$. nidulans (Table 1 ) and, equally important, successfully transferred back to $E$. coli (Figure 1).

A FAC based on an $A$. terreus SM cluster similar to a known $A$. fumigatus hexadehydroastechrome cluster [25], was chosen for HRMS screening and characterization. $A$. nidulans FAC 6J7 yielded a unique compound, the stable astechrome intermediate terezine D (Figure 3). This was consistent with the predicted metabolite production for this gene cluster (Figure 2a). Currently our focus is on elucidating novel chemistries of two other FACs which produce metabolites with masses that are not consistent with 
any known fungal metabolites (data not shown), as well as measuring mRNA levels in strains expressing FACs of interest (including Fac 9D19) to confirm that all encoded genes are being correctly expressed.

We anticipate that many alternative and expanded studies will follow on from this work. For instance, in addition to the FAC end sequencing and reference genome alignment presented in this work, there are optional and potentially more economic genomic tools to identify SM containing FACs, such as pooling FACs for either PCR or Southern hybridization-based library screening of SM backbone genes. Alternatively, extracts of FAC transformants could be first screened for desired activity and then only those showing activity be subjected to FAC sequencing. These alternative approaches could be useful for those genomes which are poorly- or not sequenced. We also envision an expansion of FAC libraries to include not only Aspergillus spp. but also other genera. The successful expression of Neurospora crassa and lichen promoters in $A$. nidulans may suggest that a fair number of Ascomycetes can be used in our system, because the lichen promoters are most likely from the fungus in the symbiotic relationship, which is typically an ascomycete $[43,44]$.

\section{Conclusions}

The results that we have described represent significant advancements to the field of translating whole genome sequence information into functional genomics and genome biology. We report the first FAC system, which is capable to shuttle and stably maintain large $(>150 \mathrm{~kb})$ DNA fragments in both E. coli and the filamentous fungus. Our concept of a large FAC equals one intact SM gene cluster including all genes and regulatory elements within a large gene cluster and/or pathway for heterologous expression provides a route for the discovery of natural products potentially missed by traditional methods. Further analysis of the entire set of the intact SM gene clusters of $A$. terreus will deepen our understanding of the dynamics of SM gene pathways and the fungal natural products.

In summary, we have successfully created a breakthrough FAC technology that can help address the challenge of characterizing the accruing fungal SM genome data. This technology allows for the creation of a SM cluster library of a single fungal species that can be shuttled and expressed in A. nidulans and likely other appropriate fungal hosts in one transformation step. This will allow the detailed genetic analysis and manipulation of fungal gene clusters from a wide range of species in the future. Additionally, we have validated an analytical and statistical pipeline to confidently identify the compound(s) encoded by the SM cluster-containing FACs, resulting in the confident discovery and identification of the astechrome precursor terezine $\mathrm{D}$ and discovery of the astechrome biosynthetic machinery in $A$. terreus.
This methodology enables the unbiased library construction of entire genomes of not only sequenced fungi but also potentially for unsequenced, and even unculturable fungi when sufficient material can be collected for DNA preparation. When combined with the high sensitivity of HRMS-based metabolomics, this technology has the potential to identify intact gene clusters and their associated SMs in fungi and other complex microbial metagenomes on a scale not previously considered feasible.

\section{Methods}

\section{Construction of shuttle BAC vectors}

To construct the E. coli and Aspergillus shuttle BAC vectors, the $A$. nidulans AMA1 gene fragment $(5.250 \mathrm{~kb})$ was blunt-ended with the DNA terminator kit (Lucigen) and cloned into the blunted-BamHI site immediately next to the Aspergillus parasiticus pyrG gene in pJW24 to form pJW24-AMA1. The $8.385 \mathrm{~kb}$ DNA fragment containing AMA1-pyrG was released from pJW24-AMA1 with NotI and BssHII double digestion, blunt-ended and cloned into the blunted-ApaI site of pSMART-BAC vector (www.lucigen.com). All reactions were performed with $100 \sim 200$ ng of each DNA with a total $30 \mu \mathrm{L}$ of reaction volume and $1 \mu \mathrm{L}$ of each enzyme; DNA was purified with the QIAGEN mini kit between each step. Due to both orientation combinations of two-step blunt-end ligations, there were four versions of the autonomous replication E. coli and Aspergillus shuttle BAC vectors: pSMARTBACpyrGAMA1-4 (Additional file 1: Figure S1a). The vector sequences were confirmed by sequencing. All shuttle vectors (FAC vectors) were successfully tested for $A$. nidulans transformation (Additional file 1: Figure S1b).

\section{Preparation of high molecular weight $A$. terreus DNA}

Aspergillus terreus strain ATCC2054 was chosen for this work. Different fungal starting materials were compared to test for quality of high molecular weight (HMW) genomic DNA: spores, germinated spores, protoplasts, or nuclei obtained from protoplasts. The protoplast preparation method has been described before [45]. To isolate nuclei, protoplasts were lysed with $0.5 \%$ Triton X-100 in HMW DNA preparation buffer (0.5 M Sucrose, $80 \mathrm{mM} \mathrm{KCl,} 10 \mathrm{mM}$ Tris, $10 \mathrm{mM}$ EDTA, $1 \mathrm{mM}$ spermidine, $1 \mathrm{mM}$ spermine, $\mathrm{pH}$ 9.4). The protoplasts in buffer were gently mixed, incubated on ice for 30 minutes, and the resulting nuclei pelleted at $1,800 \mathrm{~g}$ for 20 minutes. To prepare low melting agarose plugs of HMW DNA, the pellet $\left(\sim 5 \times 10^{8}\right)-$ be it of nuclei, protoplasts, germinated spores, or spores was resuspended with the HMW DNA preparation buffer to a total volume of $0.6 \mathrm{~mL}$, and an equal volume of $1 \%$ low melting agarose was then added to the buffer to a total volume of ca $1.2 \mathrm{~mL}$ at $45^{\circ} \mathrm{C}$. This was sufficient to make 10 plugs (about $100 \mu \mathrm{L}$ per plug) 
which solidified at $4^{\circ} \mathrm{C}$. The plugs were then incubated at $50^{\circ} \mathrm{C}$ for 48 hours in $1 \mathrm{~mL}$ lysis buffer/plug: $0.5 \mathrm{M}$ EDTA, pH 9.0, $1 \%$ lauryl sarcosine, $1 \mathrm{mg} / \mathrm{mL}$ proteinase K. Finally, the plugs were extensively washed in 10-20 volumes of the following buffers for one hour each wash: once with buffer 1 (0.5 M EDTA, pH 9.09.3 at $\left.50^{\circ} \mathrm{C}\right)$, once with buffer $2(0.05 \mathrm{M}$ EDTA, $\mathrm{pH} 8.0$ on ice), three times with buffer 3 (ice cold TE plus $0.1 \mathrm{mM}$ phenylmethyl sulfonyl fluoride (PMSF) on ice), three times with buffer 4 (ice cold TE on ice) and finally all plugs were stored in $\mathrm{TE}$ at $4^{\circ} \mathrm{C}$. In order to estimate the size and yield of extracted DNA, plugs were assessed using pulsed field gel electrophoresis (PFGE) (Bio-Rad CHEF Mapper, Hercules, CA). The final quality-check condition for the HMW genomic DNA was $6 \mathrm{~V} / \mathrm{cm}, 10 \mathrm{sec}$ to $1 \mathrm{~min}$ switch time for 12-16 hours at $14^{\circ} \mathrm{C}$ by PFGE, along with appropriate HMW size markers [46]. The highest quality and quantity of HMW genomic DNA was obtained from the protoplast preparation (Additional file 1: Figure S2a).

\section{Construction of unbiased shuttle BAC library of $A$. terreus DNA}

The HMW genomic DNA obtained from the protoplast preparation ranged from $20 \sim 200 \mathrm{~kb}$. The HMW DNA from three plugs was end-repaired with the DNA terminator kit (www.lucigen.com) in a total volume of $500 \mu \mathrm{L}$ with $10 \mu \mathrm{L}$ of the end repairing enzymes which were heat inactivated $\left(70^{\circ} \mathrm{C}, 15 \mathrm{~min}\right)$. T he resulting DNA was ligated with $B s t X \mathrm{I}$ adaptors $(10 \mu \mathrm{L}$ of $100 \mu \mathrm{M}$ each) in a total volume of $700 \mu \mathrm{L}$ consisting of a ligation reaction of $10 \mu \mathrm{L}$ ligase (2 $\mathrm{U} / \mu \mathrm{L}$, Epicenter). Gel-fractionated DNA fragments ranging from 100 to $200 \mathrm{~kb}$ were purified by PFGE. Purified large DNA fragments (about $100 \mu \mathrm{L} 1-3 \mathrm{ng} / \mu \mathrm{L}$ ) were ligated into the cloning-ready $\mathrm{BAC} B s t X \mathrm{I}$ shuttle vector (also called pSMARTBACpyrGAMA3) at $16^{\circ} \mathrm{C}$ for $\sim 18$ hours. Next, the ligated DNA mixture was electroporated into competent E. coli cells (BAC-Optimized E. coli 10G Replicator Cells, Lucigen). Small-scale ligations and transformations ( $1 \mu \mathrm{L}$ DNA per $20 \mu \mathrm{L}$ cells) were used to judge the cloning efficiency. The insert sizes of about 50 BAC clones were determined and confirmed to include inserts of about $100 \mathrm{~kb}$ (Additional file 1: Figure S2b). Once the suitability of the ligated DNA was confirmed, largescale ligations and transformations were conducted to achieve at least 7,680 clones for colony picking (20 X 384well plates) for the unbiased shuttle BAC library.

\section{BAC end sequencing, and select SM cluster-containing candidate FAC clones}

BAC-end sequences of 3,840 clones from the unbiased Random Shear BAC library of $A$. terreus were completed by the Sanger BigDye sequencing method. The software
Phred was used for base calling and sequence trimming. Vector masking was achieved using the DNAStar SeqMan Pro software package. The BAC end sequences were aligned against the $A$. terreus reference genome sequence by blastn http://www.broadinstitute.org/annotation/genome/aspergillus_group/Blast.html;jsessionid= 20A2ECF0FCDB84CC880624664797EEF8.route980?sp =Sblastn; All 56 SM clusters-containing candidate FAC clones were successfully identified based on the FAC end sequence flanking one end of a SM cluster and the other FAC end sequence flanking the other end of the same SM cluster (data not shown).

\section{Microbial strains and culture conditions}

The parental strain RJW256 (pyrG89, pyroA4, $\Delta n k u 70::$ $\operatorname{argB}, \triangle S T:: a f p y r G, v e A 1)$ was obtained by a sexual cross between LO4641 (riboB2, pyroA4, $\triangle S T:: A f p y r G, \triangle A N 79$ 09::afpyrG, $\triangle n k u 70:: \operatorname{argB}, v e A 1)$ and RJW113.5 ( $\triangle v e A::$ $\operatorname{argB}$, pyrG89). RJW256 was transformed with FAC plasmids as shown in Table 1 to produce FAC recombinant strains. $\triangle S T:: A f p y r G$ indicates that the entire endogenous sterigmatocystin gene cluster was removed from $A$. nidulans.

For antimicrobial activity tests, we used $A$. nidulans RDIT9.32, A. fumigatus 293, Candida albicans, Pseudomonas aeroginosa PAO1, Bacillus cereus U85, and Micrococcus luteus strains. All of the fungal and bacterial strains were maintained as frozen glycerol stocks at $-80^{\circ} \mathrm{C}$. Fungal strains were grown at $37^{\circ} \mathrm{C}$ on glucose minimal medium (GMM, [45]) and bacterial strains were cultured on tryptic soy broth medium.

\section{A. nidulans transformation and the recovery of SM cluster-containing FACs}

A modified PEG-calcium based transformation method was applied to improve transformation yield because our published methods [45] did not work well with the $100 \mathrm{~kb}$ FAC vectors. The method was modified as follows: $200 \mu \mathrm{L}$ containing $10^{7} \mathrm{~A}$. nidulans RJW256 protoplasts mixed with $2 \mu \mathrm{g}$ FAC DNA was gently placed over $200 \mu \mathrm{L}$ of $30 \%$ PEG 4,000 with $50 \mathrm{mM} \mathrm{CaCl}_{2}$ in $1.5 \mathrm{~mL}$ centrifuge tube. The centrifuge tube with protoplasts was incubated $30 \mathrm{~min}$ on ice. After centrifuging the incubated mixture for $5 \mathrm{~min}$ at $250 \times \mathrm{g}$, the solution was gently mixed using an autopipette. This mixture was then incubated for $10 \mathrm{~min}$ at room temperature before $1 \mathrm{~mL}$ of sorbitol-Tris-HCl-CaCl${ }_{2}$ (STC: $1.2 \mathrm{M}$ sorbitol, $10 \mathrm{mM}$ Tris- $\mathrm{HCl}, 10 \mathrm{mM} \mathrm{CaCl} 2 \mathrm{pH7.5)}$ buffer was added and gently mixed into the solution. After transferring the mixture into a $13 \mathrm{~mL}$ tube, an additional $5 \mathrm{~mL}$ of STC was added into the tube and gently mixed. One $\mathrm{mL}$ of this final solution was distributed onto regeneration media to obtain transformants. 
A. nidulans FAC transformants (Table 1) were maintained on culture plates for three generations for phenotype and chemical screening. For FAC recovery, we prepared $\sim 0.3 \mathrm{~mL}$ of $10^{6} / \mathrm{mL}$ protoplasts from $A$. nidulans FAC strains and FAC DNA was isolated by the common alkali lysis method, and resuspended in $10 \mu \mathrm{L}$ of TE. One microliter of recovered DNA was retransformed back into $E$. coli cells (BAC-Optimized $E$. coli 10G Replicator Cells, Lucigen).

\section{Fungal genomic DNA extraction}

Fungal DNA was extracted from lyophilized mycelia using previously described techniques [47] to perform PCR reaction with primers listed in Additional file 3: Table S2.

\section{Antimicrobial screening}

A disc-diffusion method [48] was used for antibiotic activity-guided screening. One plate per each $A$. nidulans FAC strain was inoculated on solid GMM and incubated for seven days at $37^{\circ} \mathrm{C}$. Subsequently, the entire contents of the plates were collected and lyophilized for 48 hours. Samples were then pulverized with mortar and pestle prior to the addition of $10 \mathrm{~mL}$ of methanol. Air-dried methanol extracts were dissolved in $150 \mu \mathrm{L}$ methanol for activity testing. Media preparation for antibacterial assays were performed as previously described [45]. For antifungal assays, $10^{6}$ spores mentioned in the section above were embedded in $5 \mathrm{~mL}$ soft GMM agar (0.75\% agar) and overlaid on solid GMM. Ten $\mu \mathrm{L}$ out of the $150 \mu \mathrm{L}$ methanol extract above was loaded on a $1 \mathrm{~cm}$ diameter paper disc for each assay. Assay plates were incubated for 24 to 48 hour at $37^{\circ} \mathrm{C}$ and observed for antimicrobial activity.

\section{LC-HRMS analysis}

Five plates for $A$. nidulans FAC strain 6J7 were inoculated on solid GMM and incubated for seven days at $37^{\circ} \mathrm{C}$. Subsequently, the entire contents of the plates were collected and lyophilized for 48 hours. Samples were then pulverized with mortar and pestle prior to the addition of $10 \mathrm{~mL}$ of methanol. Air-dried methanol extracts were then further extracted with organic solvent (chloroform:methanol:ethylacetate $=8: 1: 1$ ). Organic extracts were evaporated to dryness and stored at $-20^{\circ} \mathrm{C}$ until analysis.

Organic extracts obtained were resuspended in methanol to a final concentration of $2 \mu \mathrm{g} / \mu \mathrm{L}$. For each analysis, $40 \mu \mathrm{g}$ of sample was loaded onto a Luna C18 column $(150 \mathrm{~mm} \times 2 \mathrm{~mm} ; 3 \mu \mathrm{m}$ particle size) (Phenomenex, Torrance, CA). Chromatography was performed using an Agilent 1150 LC system (Agilent, Santa Clara, CA) at a flow rate of $200 \mu \mathrm{L} / \mathrm{min}$. The following gradient was employed (Buffer A: water with $0.1 \%$ formic acid, Buffer B: acetonitrile with $0.1 \%$ formic acid): time $0 \mathrm{~min}$, $2 \% \mathrm{~B} ; 35 \mathrm{~min}, 70 \% \mathrm{~B}$; $54 \mathrm{~min}, 98 \%$ B. A 1:7 split was employed post-column, resulting in a flow rate of $25 \mu \mathrm{L} /$ min being directed to the mass spectrometer. A QExactive mass spectrometer (Thermo Fisher Scientific, Waltham, MA) was used for MS analysis with the following settings: capillary temperature $275^{\circ} \mathrm{C}$, sheath gas 4 (arbitrary units), spray voltage $4.2 \mathrm{kV}$. Full MS spectra were acquired at 35,000 resolution for the mass range $\mathrm{m} / z 200$ to 1500 for all samples. Following each full MS scan, the top 5 most intense ions were selected for a dependent $\mathrm{MS}^{2}$ scan. $\mathrm{MS}^{2}$ was conducted using higherenergy collisional dissociation (HCD) with a normalized collision energy of $30 \%$. Three biological replicates of FAC 6J7 extracts were prepared and analyzed in technical duplicate, followed by the data workup described below.

\section{Data analysis, informatics, and software}

The SIEVE software suite (Thermo Fisher Scientific, Waltham, MA) was used for component detection and relative quantification of ions produced by electrospray during small molecule LC-HRMS. Component detection was performed using a mass tolerance of 10 part-permillion (ppm) and a retention time window of $2.5 \mathrm{~min}$. A minimum intensity of $5 \times 10^{6}$ was selected as the threshold for defining a peak as a component. For each component, a selected ion chromatogram was created and the integrated intensity of the peak was calculated. Peak areas were normalized based on total ion current. To increase statistical power and confidence of the final analysis, the procedure adopted here involved a decoy approach to multiple hypothesis testing. Specifically, the replicate data FAC 6J7 was subjected to a uniqueness filter against processed LC-HRMS data generated from a control group of strains containing empty vectors, as well as 13 other strains containing a variety of other FACs with unique genetic content. For dereplication, all components were initially searched against a targeted accurate mass database consisting of known fungal metabolites produced by $A$. nidulans and $A$. terreus using a mass tolerance of $3 \mathrm{ppm}$. A dozen of these known compounds were present at consistent levels in nearly all samples, and were monitored to rapidly identify highly perturbed systems. All components were also searched against a comprehensive accurate mass database consisting of over 13,000 known fungal secondary metabolites. This fungal database was prepared using Antibase [49], Dictionary of Natural Products [50], as well as additional fungal natural products found in the literature [51,52].

\section{Additional files}

Additional file 1: Figure S1. Panel a: FAC vectors: pSMARTBACpyrGAMA1 $\sim 4$, each has two Not I sites (N) flanking the cloning site (B, Bst XI) within the BAC end sequencing primers SP6 and T7. In addition to 
the chloramphenicol resistance gene (camR), loxP and cos sites, pyrGA represents the pyrG gene from Aspergillus parasiticus, AMA1 is the replication origin of fungal artificial chromosome, genes parA and parB are for active partitioning and gene sopC is to ensure that each daughter cell gets a copy of the shuttle BAC plasmid, gene repE is for BAC plasmid replication and regulation of copy number, and oriV is the BAC replication origin. Panel b: A. nidulans FAC transformants using PSMARTBACPyrGAMA3 vector. Figure S2. Preparation of HMW genomic DNA from $A$. terreus and random shear FAC cloning results. Panel a: A. terreus HMW genomic DNA ranging from 20-200 kb. Panel b: CHEF gel electrophoresis and Notl digestion of random selected FAC clones, the average insert size was estimated at $\sim 110 \mathrm{~kb} . \mathrm{M}$, Lambda ladder Marker. Figure S3. Three additional CHEF gels of E. coli-Aspergillus shuttle FACs that were successfully transferred from transformed strains of A. nidulans back into $E$. coli. The examples of recovered FAC clones shown here include 903 (cluster 30, 100 kb), $9 \mathrm{~A} 23$ (cluster $25, \sim 80 \mathrm{~kb}$ ), and 7A10 (cluster $56, \sim 90 \mathrm{~kb}$ ) from top to bottom panel. The first and last lanes are DNA Lambda ladder Markers, the $2^{\text {nd }}$ and $3^{\text {rd }}$ lane(s) on the left hand side of the gels is the control FAC used to transform A. nidulans, and all of other lanes are randomly selected FAC clones recovered. All control and recovered FACs were digested with Not I. Figure S4. Antibiotic activity test of 14 FAC clones. Ten $\mu \mathrm{L}$ out of $150 \mu \mathrm{L}$ methanol extract from FAC transformants cultured on GMM plate for 7 days at $37^{\circ} \mathrm{C}$ was loaded on small disc (diameter: $1 \mathrm{~cm}$ ) for antimicrobial activity test against Aspergillus spp., Candida albicans, Bacillus cereus, Micrococcus luteus and Pseudomonas aeruginosa. Antibiotic activity was observed against Bacillus cereus with two FAC extracts.

Additional file 2: Table S1. BAC clones covering 56 SM clusters identified by both BAC end sequences.

Additional file 3: Table S2 PCR primers used in this study.

\section{Competing interests}

C.C.W. and R.Y. are employees of Intact Genomics Inc., a company that sells the unbiased Random Shear BAC libraries and services for genome discovery and DNA research. D.M., M.W. and A.K. are employees of Lucigen Corporation, a company that sells BAC cloning, DNA end repairing, and other enzyme kits for DNA and protein research.

\section{Authors' contributions}

CCW and NPK conceived and supervised the project. DM participated in the SBIR Phase I grant writing, provided advice during this discovery research, and edited this manuscript. The shuttle FAC vectors and the unbiased Random Shear FAC library were constructed by CCW and RY The FAC end sequencing, analysis, FAC DNA preparation, and the identification of secondary metabolic pathway-containing FAC clones were performed by $C C W, R Y, M W$, and AK. The shuttle FACs were recovered and characterized by RY and JWB. JWB performed the A. nidulans transformation with FACs, their characterization, and prepared samples for metabolite identification and structure determination. AWG prepared samples for LC-MS analysis and collected LC-MS data. KDC conducted analysis of LC-MS data, including development of the analysis pipeline, compound identification, and structure elucidation under the supervision of PMT and NLK. The manuscript was prepared by NPK, CCW, JWB, KDC, JCA, RY, PMT, and NLK. All authors read and approved the final manuscript.

\section{Acknowledgments}

We thank Berl Oakley for supplying us with strain LO4641. This work was supported in part by National Institutes of Health 1R43Al94885-1 to C.C.W. at Lucigen Corporation and to N.P.K, R01GM067725 to N.L.K., 5PO1GM084077 to N.P.K., and Innovation \& Economic Development Research 101PRJ72KQ to N.P.K.

\section{Author details}

'Department of Medical Microbiology and Immunology and Bacteriology, University of Wisconsin at Madison, Madison, WI, USA. ${ }^{2}$ Intact Genomics, Inc., St Louis, MO, USA. ${ }^{3}$ Proteomics Center of Excellence, Northwestern University, Evanston, IL, USA. ${ }^{4}$ Lucigen Corporation, Middleton, WI, USA. ${ }^{5}$ Department of Chemistry, Northwestern University, Evanston, IL, USA. ${ }^{6}$ Department of Molecular Biosciences, Northwestern University, Evanston, IL, USA.
Received: 26 December 2014 Accepted: 20 April 2015 Published online: 29 April 2015

\section{References}

1. Blackwell M. The fungi: 1, 2, 3 . . 5.1 million species? Am J Bot. 2011;98:426-38.

2. Inglis DO, Binkley J, Skrzypek MS, Arnaud MB, Cerqueira GC, Shah P, et al. Comprehensive annotation of secondary metabolite biosynthetic genes and gene clusters of Aspergillus nidulans, A. fumigatus, A. niger and A. oryzae. BMC Microbiol. 2013;13:91.

3. Grigoriev IV, Cullen D, Goodwin SB, Hibbett D, Jeffries TW, Kubicek CP, et al. Fueling the future with fungal genomics. Mycology. 2011;2:192-209.

4. Brakhage AA, Schroeckh V. Fungal secondary metabolites - strategies to activate silent gene clusters. Fungal Genet Biol. 2011;48:15-22.

5. Strauss J, Reyes-Dominguez Y. Regulation of secondary metabolism by chromatin structure and epigenetic codes. Fungal Genet Biol. 2011:48:62-9.

6. Hong SY, Roze LV, Linz JE. Oxidative stress-related transcription factors in the regulation of secondary metabolism. Toxins (Basel). 2013;5:683-702.

7. Palmer JM, Keller NP. Secondary metabolism in fungi: does chromosomal location matter? Curr Opin Microbiol. 2010;13:431-6.

8. Itoh T, Kushiro T, Fujii I. Reconstitution of a secondary metabolite biosynthetic pathway in a heterologous fungal host. Methods Mol Biol. 2012;944:175-82.

9. Chiang YM, Oakley CE, Ahuja M, Entwistle R, Schultz A, Chang SL, et al. An efficient system for heterologous expression of secondary metabolite genes in Aspergillus nidulans. J Am Chem Soc. 2013;135:7720-31.

10. Nielsen MT, Nielsen JB, Anyaogu DC, Holm DK, Nielsen KF, Larsen TO, et al. Heterologous reconstitution of the intact geodin gene cluster in Aspergillus nidulans through a simple and versatile PCR based approach. PLoS One. 2013;8, e72871.

11. Tsunematsu Y, Ishiuchi K, Hotta K, Watanabe K. Yeast-based genome mining, production and mechanistic studies of the biosynthesis of fungal polyketide and peptide natural products. Nat Prod Rep. 2013;30:1139-49.

12. Yin WB, Chooi YH, Smith AR, Cacho RA, Hu Y, White TC, et al. Discovery of cryptic polyketide metabolites from Dermatophytes using heterologous expression in Aspergillus nidulans. ACS Synth Biol. 2013;2:629-34.

13. Zhu H, Choi S, Johnston AK, Wing RA, Dean RA. A large-insert (130 kbp) bacterial artificial chromosome library of the rice blast fungus Magnaporthe grisea: genome analysis, contig assembly, and gene cloning. Fungal Genet Biol. 1997;21:337-47.

14. Nishimura M, Nakamura S, Hayashi N, Asakawa S, Shimizu N, Kaku H, et al. Construction of a BAC library of the rice blast fungus Magnaporthe grisea and finding specific genome regions in which its transposons tend to cluster. Biosci Biotechnol Biochem. 1998;62:1515-21.

15. Adler $\mathrm{H}$, Messerle $\mathrm{M}$, Koszinowski UH. Cloning of herpesviral genomes as bacterial artificial chromosomes. Rev Med Virol. 2003;13:111-21.

16. Diener SE, Chellappan MK, Mitchell TK, Dunn-Coleman N, Ward M, Dean RA. Insight into Trichoderma reesei's genome content, organization and evolution revealed through BAC library characterization. Fungal Genet Biol. 2004;41:1077-87.

17. Srivastava SK, Huang X, Brar HK, Fakhoury AM, Bluhm BH, Bhattacharyya MK. The genome sequence of the fungal pathogen Fusarium virguliforme that causes sudden death syndrome in soybean. PLoS One. 2014;9, e81832.

18. Béjà O. To BAC or not to BAC: marine ecogenomics. Curr Opin Biotechnol. 2004;15:187-90,

19. Lorenz P, Eck J. Metagenomics and industrial applications. Nat Rev Microbiol. 2005;3:510-6.

20. Ongley SE, Bian X, Neilan BA, Müller R. Recent advances in the heterologous expression of microbial natural product biosynthetic pathways. Nat Prod Rep. 2013;30:1121-38.

21. Godiska R, Mead DA, Dhodda V, Hochstein R, Karsi A, Ravin N, et al. Bias-Free Cloning of 'Unclonable' DNA for Simplified Genomic Finishing. In DNA Sequencing III: Dealing with Difficult Templates. Sudbury, MA: Jones and Bartlett Publishers; 2008.

22. Aleksenko A, Clutterbuck A. Autonomous plasmid replication in Aspergillus nidulans: AMA1 and MATE elements. J Fungal Genet Biol. 1997;21:373-87.

23. Zhang HB, Scheuring CF, Zhang M, Zhang Y, Wu CC, Dong JJ, et al. Construction of BIBAC and BAC libraries from a variety of organisms for advanced genomics research. Nat Protoc. 2012;7:479-99. 
24. Khaldi N, Seifuddin FT, Turner G, Haft D, Nierman WC, Wolfe KH, et al. SMURF: Genomic mapping of fungal secondary metabolite clusters. Fungal Genet Biol. 2010;47:736-41.

25. Yin WB, Baccile JA, Bok JW, Chen Y, Keller NP, Schroeder FC. A nonribosomal peptide synthetase-derived iron(III) complex from the pathogenic fungus Aspergillus fumigatus. J Am Chem Soc. 2013;135:2064-7.

26. Watanabe T, Arisawa M, Narusuye K, Alam MS, Yamamoto K, Mitomi M, et al. Alantrypinone and its derivatives: synthesis and antagonist activity toward insect GABA receptors. Bioorg Med Chem. 2009;17:94-111.

27. Kobayashi A, Hino T, Yata S, Itoh TJ, Sato H, Kawazu K. Unique spindle poisons, curvularin and its derivatives, isolated from Penicillium species. Agric Biol Chem. 1988;52:3119-23.

28. Kuno F, Otoguro K, Shiomi K, Iwai Y, Omura S. Arisugacin A and B, novel and selective acetylcholinesterase inhibitors from Penicillium sp. FO 4259. I. Screening, taxonomy, fermentation, isolation and biological activity. J Antibiot (Tokyo). 1996;49:742-7.

29. Kumar CG, Mongolla P, Pombala S, Kamle A, Joseph J. Physicochemica characterization and antioxidant activity of melanin from a novel strain of Aspergillus bridgeri ICTF-201. Lett Appl Microbiol. 2011;53:350-8.

30. Wu MC, Law B, Wilkinson B, Micklefield J. Bioengineering natural product biosynthetic pathways for therapeutic applications. Curr Opin Biotechnol. 2012;23:931-40.

31. Du L, Robles AJ, King JB, Powell DR, Miller AN, Mooberry SL, et al. Crowdsourcing natural products discovery to access uncharted dimensions of fungal metabolite diversity. Angew Chem Int Ed Engl. 2014;53:804-9.

32. Fang SM, Wu CJ, Li CW, Cui CB. A practical strategy to discover new antitumor compounds by activating silent metabolite production in fungi by diethyl sulphate mutagenesis. Mar Drugs. 2014;12:1788-814.

33. Leitão AL, Enguita FJ. Fungal extrolites as a new source for therapeutic compounds and as building blocks for applications in synthetic biology. Microbiol Res. 2014;169:652-65.

34. Takken FL, Van Wijk R, Michielse CB, Houterman PM, Ram AF, Cornelissen $B J$. One-step method to convert vectors into binary vectors suited for Agrobacterium-mediated transformation. Curr Genet. 2004;45:242-8.

35. Aleksenko A, Makarova N, Nikolaev I, Clutterbuck AJ. Integrative and replicative transformation of Penicillium canescens. Curr Genet. 1995:28:474-7.

36. Aleksenko A, Gems D, Clutterbuck J. Multiple copies of MATE elements support autonomous plasmid replication in Aspergillus nidulans. Mol Microbiol. 1996;20:427-34.

37. Aleksenko A, Nikolaev I, Vinetski Y, Clutterbuck AJ. Gene expression from replicating plasmids in Aspergillus nidulans. Mol Gen Genet. 1996;253:242-6.

38. Fierro F, Kosalkova K, Gutierrez S, Martin JF. Autonomously replicating plasmids carrying the AMA1 region in Penicillium chrysogenum. Curr Genet. 1996:29:482-9.

39. Liu W, May GS, Lionakis MS, Lewis RE, Kontoyiannis DP. Extra copies of the Aspergillus fumigatus squalene epoxidase gene confer resistance to terbinafine: genetic approach to studying gene dose-dependent resistance to antifungals in A. fumigatus. Antimicrob Agents Chemother. 2004;48:2490-6.

40. Xue T, Nguyen CK, Romans A, Kontoyiannis DP, May GS. Isogenic auxotrophic mutant strains in the Aspergillus fumigatus genome reference strain AF293. Arch Microbiol. 2004;182:346-53.

41. Shizuya H, Birren B, Kim U-J, Mancino V, Slepak T, Tachiiri Y, et al. Cloning and stable maintenance of 300-kilobase-pair fragments of human DNA in Escherichia coli using an F-factor-based vector. Proc Natl Acad Sci U S A. 1992:89:8794-7.

42. Murray AW, Szostak JW. Construction of artificial chromosomes in yeast. Nature. 1983;305:189-93.

43. Bird D, Bradshaw R. Gene targeting is locus dependent in the filamentous fungus Aspergillus nidulans. Mol Gen Genet. 1997;255:219-25.

44. Sinnemann SJ, Andrésson OS, Brown DW, Miao VP. Cloning and heterologous expression of Solorina crocea pyrG. Curr Genet. 2000;37:333-8.

45. Bok JW, Keller NP. LaeA, a regulator of secondary metabolism in Aspergillus spp. Eukaryotic Cell. 2004;3:527-35.

46. Zhang M, Zhang Y, Scheuring CF, Wu CC, Dong JJ, Zhang HB. Preparation of megabase-sized DNA from a variety of organisms using the nuclei method for advanced genomics research. Nat Protoc. 2012;7:467-78.

47. Bok JW, Keller NP. Fast and easy method for construction of plasmid vectors using modified quick-change mutagenesis. Methods Mol Biol. 2012;944:163-74.

48. Bauer AW, Kirby WM, Sherris JC, Turck M. Antibiotic susceptibility testing by a standardized single disk method. Am J Clin Pathol. 1966;45:493-6.

49. Laatsch, H. Antibase 2011; Wiley VCH: Weinheim, Germany, 2011
50. Running, W. E. (1993) Chapman and Hall Dictionary of Natural-Products on Cd-Rom. J. Chem. Inf. Comput. Sci. 33, 934-935.

51. Caboche $S$, Pupin $M$, Leclère $V$, Fontaine $A$, Jacques $P$, Kucherov G. NORINE: a database of nonribosomal peptides. Nucleic Acids Res. 2008;36:D326-31.

52. Andersen MR, Nielsen JB, Klitgaard A, Petersen LM, Zachariasen M, Hansen $\mathrm{TJ}$, et al. Accurate prediction of secondary metabolite gene clusters in filamentous fungi. Proc Natl Acad Sci U S A. 2013;110:E99-107.

\section{Submit your next manuscript to BioMed Central and take full advantage of:}

- Convenient online submission

- Thorough peer review

- No space constraints or color figure charges

- Immediate publication on acceptance

- Inclusion in PubMed, CAS, Scopus and Google Scholar

- Research which is freely available for redistribution

Submit your manuscript at www.biomedcentral.com/submit 